\title{
A theorem of H. Hopf and the Cauchy-Riemann inequality II
}

\section{Hilário Alencar, Manfredo do Carmo, Isabel Fernández* and Renato Tribuzy}

\begin{abstract}
This is a sequel to "A theorem of H. Hopf and the Cauchy-Riemann inequality" [AdCT]. Here the result of the previous paper is extended (see the precise statement in Section 1 of the present paper) to surfaces in three-dimensional homogeneous Riemannian manifolds whose group of isometries has dimension four and the bundle curvature is nonzero, whereas in the previous paper only the case of vanishing bundle curvature was treated.
\end{abstract}

Keywords: mean curvature, genus zero surface, Hopf's quadratic form, CauchyRiemann inequality.

Mathematical subject classification: Primary: 53C42; Secondary: 20A10.

\section{Introduction}

Let $\mathbb{E}^{3}$ be a 3-dimensional simply-connected homogeneous riemannian manifold with a 4-dimensional isometry group. Such a manifold is a riemannian fibration with bundle curvature $\tau$ over a 2-dimensional space form with sectional curvature $k$. They are classified, up to isometries, by $k$ and $\tau$, where $k$ and $\tau$ are any two real numbers with $k \neq 4 \tau^{2}$ and $k^{2}+\tau^{2} \neq 0$. We willdenote

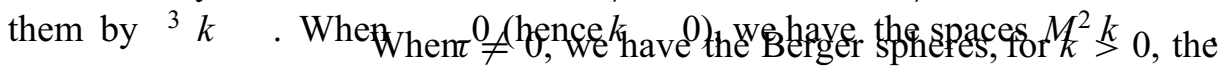

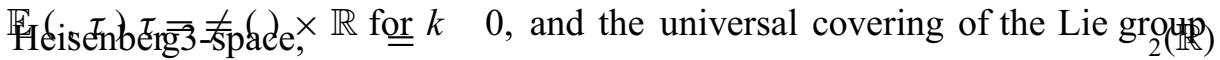
$S L<$ for $k \quad 0$. Theibers of ${ }^{3} k$ are geodesics (straight lines in the case $0)$ thetrans- $\mathbb{E}(, \tau) \tau=$ lations along which generate a Killing vector field $\xi$. The bundle curvature $\tau$ is given by $\nabla_{X} \xi=\tau \xi \times X$, where $\nabla$ is the covariant derivative, $X$ is any vector $\mathrm{R}$ 
field, and $\times$ is the cross product in $\mathbb{E}^{3}(k, \tau)$. We refer to [D] for further details on these spaces and their immersed surfaces.

For the case $\tau=0$, Abresch and Rosenberg [AR1] considered a surface $M$ immersed in $M^{2}(k) \times \mathbb{R}$ and introduced a quadratic form on $M$ by

$$
Q(X, Y)=2 H \alpha(X, Y)-k\langle\xi, X\rangle\langle\xi, Y\rangle,
$$

where $X$ and $Y$ are tangent vectors, $\alpha$ is the second fundamental form and $H$ is the mean curvature of $M$. Introduce in $M$ isothermal parameters $(u, v)$ (this means that the induced metric on $M$ is $\left.d s^{2}=\lambda^{2}\left(d u^{2}+d v^{2}\right)\right)$ and let $z=u+i v$ be the corresponding complex parameter.

Abresch and Rosenberg proved the $(2,0)$-component $Q^{(2,0)}$ of $Q$ is holomorphic if $H=$ const. This generalizes a previous result of $\mathrm{H}$. Hopf $[\mathrm{H}]$ for the case where $M$ is immersed in $\mathbb{R}^{3}$. Furthermore, they showed that if $M$ is homeomorphic to a sphere, then $M$ is an embedded surface invariant by rotations of $M^{2}(k) \times \mathbb{R}$ around the factor $\mathbb{R}$.

Alencar, do Carmo and Tribuzy [AdCT] showed that it is not necessary to assume that $H=$ const. in the above result. They proved the following result

Theorem A. Let $M$ be a compact immersed surface of genus zero in $M^{2}(k) \times \mathbb{R}$. Assume that

$$
|d H| \leq g\left|Q^{(2,0)}\right|,
$$

where $|d H|$ is the norm of the differential $d H$ of the mean curvature $H$ of $M$, and $g$ is a continuous, nonnegative real function. Then $Q^{(2,0)}$ is identically zero, hence by [AR1], $M$ is a CMC (constant mean curvature) embedded surface invariant by rotations in $M^{2}(k) \times \mathbb{R}$.

The goal of the present paper is to generalize Theorem A for immersed surfaces $M$ in $\mathbb{E}^{3}(k, \tau)$, for $\tau \neq 0$.

For that, we first introduce the quadratic form

$$
Q(X, Y)=2(H+i \tau) \alpha(X, Y)-\left(k-4 \tau^{2}\right)\langle\xi, X\rangle\langle\xi, Y\rangle
$$

for tangent vectors $X, Y$ in $M$. The expression of the above form was inspired in a joint paper of Abresch and Rosenberg [AR2] in which they announce that the theorem of [AR1] (case $H=$ const.) can be extended to surfaces immersed in $\mathbb{E}^{3}(k, \tau)$. As usual, we denote by $Q^{(2,0)}$ the $(2,0)$-component of $Q$ in the complex structure of $M$ determined by the induced metric. We prove

Theorem 1. Let $M$ be a compact surface of genus zero immersed in $\mathbb{E}^{3}(k, \tau)$ with mean curvature $H$. Assume that

$$
|d H| \leq g\left|Q^{(2,0)}\right|,
$$


where $g$ is a continuous nonnegative real function. Then $Q^{(2,0)}$ is identically zero and, by [AR2], $M$ is a CMC surface invariant by rotations in $\mathbb{E}^{3}(k, \tau)$.

\section{Preliminaries}

Set $\theta=H+i \tau, c=k-4 \tau^{2}$ and write $Q$ as

$$
Q(X, Y)=2 \theta \alpha(X, Y)-c\langle\xi, X\rangle\langle\xi, Y\rangle .
$$

The (2,0)-component of $Q$ is

$$
Q^{(2,0)}=\psi(z) d z d z .
$$

Here $z=u+i v$, where $(u, v)$ are isothermal parameters in $M$, i.e.,

$$
\left\langle\frac{\partial}{\partial u}, \frac{\partial}{\partial u}\right\rangle=\left\langle\frac{\partial}{\partial v}, \frac{\partial}{\partial v}\right\rangle=\lambda^{2}, \quad\left\langle\frac{\partial}{\partial u}, \frac{\partial}{\partial v}\right\rangle=0,
$$

and

$$
d z=\frac{1}{\sqrt{2}}(d u+i d v), \quad d \bar{z}=\frac{1}{\sqrt{2}}(d u-i d v) .
$$

Set

$$
Z=\frac{1}{\sqrt{2}}\left(\frac{\partial}{\partial u}-i \frac{\partial}{\partial v}\right), \quad \bar{Z}=\frac{1}{\sqrt{2}}\left\langle\frac{\partial}{\partial u}+i \frac{\partial}{\partial v}\right\rangle
$$

so that

$$
d z(Z)=1=d \bar{z}(\bar{Z}), \quad d z(\bar{Z})=d \bar{z}(Z)=0 .
$$

Notice also that

$$
Q(Z, Z)=\psi(z) \quad \text { and } \quad\langle Z, \bar{Z}\rangle=\lambda^{2}, \quad\langle Z, Z\rangle=\langle\bar{Z}, \bar{Z}\rangle=0 .
$$

We first prove the lemma below that will be used repeatedly in this section.

Lemma 1. With the above notation

$$
\nabla_{\bar{Z}} Z=\nabla_{Z} \bar{Z}=0
$$

where $\nabla$ is the covariant derivative in $M$.

Proof. From the symmetry of the conexion we have

$$
\nabla_{\bar{Z}} Z-\nabla_{Z} \bar{Z}=[Z, \bar{Z}] .
$$


A straightforward computation shows that, for any function $f$ on $M$, $[Z, \bar{Z}](f)=0$, so that $\nabla_{\bar{Z}} Z=\nabla_{Z} \bar{Z}$. Now set

$$
\nabla_{\bar{Z}} Z=a Z+b \bar{Z}=\nabla_{Z} \bar{Z} \text {. }
$$

Then, since $\langle Z, Z\rangle=0$,

$$
0=\frac{1}{2} \bar{Z}\langle Z, Z\rangle=\left\langle\nabla_{\bar{Z}} Z, Z\right\rangle=b \lambda^{2} .
$$

It follows that $b=0$ and $\nabla_{\bar{Z}} Z=a Z$. Similarly, since $\langle\bar{Z}, \bar{Z}\rangle=0$,

$$
0=\frac{1}{2} Z\langle\bar{Z}, \bar{Z}\rangle=\left\langle\nabla_{Z} \bar{Z}, \bar{Z}\right\rangle=a \lambda^{2}
$$

so that $a=0$ and $\nabla_{\bar{Z}} Z=\nabla_{Z} \bar{Z}=0$.

Next, we compute

$$
\frac{d \psi}{d \bar{z}}=\bar{Z} Q(Z, Z)=\bar{Z}\left(2 \theta\langle S Z, Z\rangle-c\langle\xi, Z\rangle^{2}\right),
$$

where $S$ is the shape operator corresponding to $\alpha$.

Proposition 1. $\bar{Z} Q(Z, Z)=2 \bar{Z}(H) \alpha(Z, Z)+2 \theta \lambda^{2} Z(H)$.

Proof.

$$
\begin{aligned}
\bar{Z} Q(Z, Z)= & 2 \bar{Z}(\theta)\langle S Z, Z\rangle+2 \theta\left\langle\nabla_{\bar{Z}}(S Z), Z\right\rangle-2 c\langle\xi, Z\rangle\left\langle\bar{\nabla}_{\bar{Z}} \xi, Z\right\rangle \\
& -2 c\langle\xi, Z\rangle\left\langle\xi, \bar{\nabla}_{\bar{Z}} Z\right\rangle,
\end{aligned}
$$

where we have used that $\nabla_{\bar{Z}} Z=0$. In the Lemmas below, we compute separately $\left\langle\nabla_{\bar{Z}}(S Z), Z\right\rangle,\left\langle\bar{\nabla}_{\bar{Z}} \xi, Z\right\rangle$ and $\left\langle\xi, \bar{\nabla}_{\bar{Z}} \bar{Z}\right\rangle$.

Lemma 2. $\left\langle\nabla_{\bar{Z}}(S Z), Z\right\rangle=\left\langle\nabla_{Z}(S \bar{Z}), Z\right\rangle+c \lambda^{2}\langle\xi, N\rangle\langle\xi, Z\rangle$.

Proof. By using that $\nabla_{\bar{Z}} Z=0$ and Codazzi equation, we obtain

$$
\begin{aligned}
\nabla_{\bar{Z}}(S Z) & =\left(\nabla_{\bar{Z}} S\right)(Z)+S\left(\nabla_{Z} \bar{Z}\right) \\
& =\left(\nabla_{Z} S\right)(\bar{Z})+\widetilde{R}(Z, \bar{Z}) N \\
& =\left(\nabla_{Z} S\right) \bar{Z}+c\langle N, \xi\rangle(\langle Z, \xi\rangle \bar{Z}-\langle\bar{Z}, \xi\rangle Z),
\end{aligned}
$$

where $\widetilde{R}$ is the curvature of $\mathbb{E}^{3}(k, \tau)$ and we have used Corollary 3.2 of Daniel [D]. Finally, since $\langle Z, Z\rangle=0$, we conclude that

$$
\left\langle\nabla_{\bar{Z}}(S Z), Z\right\rangle=\left\langle\nabla_{Z}(S \bar{Z}), Z\right\rangle+c\langle N, \xi\rangle\langle Z, \xi\rangle \lambda^{2} .
$$


Lemma 3. $\left\langle\bar{\nabla}_{\bar{Z}} \xi, Z\right\rangle=i \tau \lambda^{2}\langle\xi, N\rangle$, where $N$ is the normal to the surface $M$. Proof. By using ([D], proof of the Proposition 3.2) we obtain

$$
\bar{\nabla}_{\bar{Z}} \xi=\tau \xi \times \bar{Z}=\tau(\langle J \bar{Z}, \xi\rangle N-\langle\xi, N\rangle J \bar{Z}),
$$

where $J$ is the complex multiplication by $i$. Thus

$$
\left\langle\bar{\nabla}_{\bar{Z}} \xi, Z\right\rangle=-\tau\langle\xi, N\rangle i \lambda^{2}
$$

Lemma 4. $\left\langle\xi, \bar{\nabla}_{\bar{Z}} Z\right\rangle=\lambda^{2} H\langle\xi, N\rangle$.

Proof. $\bar{\nabla}_{\bar{Z}} Z=\nabla_{\bar{Z}} Z+\alpha(\bar{Z}, Z) N$.

Since $\nabla_{Z} Z=0$ and $\alpha(\bar{Z}, Z)=\lambda^{2} H$. (See the proof of Lemma 2 in [AdCT]), we conclude that

$$
\left\langle\xi, \bar{\nabla}_{Z} Z\right\rangle=\lambda^{2} H\langle\xi, N\rangle .
$$

Putting Lemmas 2, 3 and 4 in the expression (1) of $\bar{Z} Q(Z, Z)$, we obtain

$$
\begin{aligned}
\bar{Z} Q(Z, Z)= & 2 \bar{Z}(H) \alpha(Z, Z)+2 \theta\left\langle\nabla_{Z}(S \bar{Z}), Z\right\rangle+2 \theta c \lambda^{2}\langle N, \xi\rangle\langle Z, \xi\rangle \\
& -2 c i \tau \lambda^{2}\langle\xi, Z\rangle\langle\xi, N\rangle-2 c \lambda^{2} H\langle\xi, N\rangle\langle\xi, Z\rangle .
\end{aligned}
$$

Since $\theta=H+i \tau$, the two last terms of the above sum cancel out with the third term. Thus

$$
\bar{Z} Q(Z, Z)=2 \bar{Z}(H) \alpha(Z, Z)+2 \theta\left\langle\nabla_{Z}(S \bar{Z}), Z\right\rangle .
$$

To conclude the proof of Proposition 1, we still need some information on the term $\left\langle\nabla_{Z}(S \bar{Z}), Z\right\rangle$.

Lemma 5. $\left\langle\nabla_{Z}(S \bar{Z}), Z\right\rangle=\lambda^{2} Z(H)$.

Proof. We first claim that

$$
\nabla_{Z} Z=\frac{Z\left(\lambda^{2}\right)}{\lambda^{2}} Z
$$

To see that, set $\nabla_{Z} Z=a Z+b \bar{Z}$. Then

$$
\left\langle\nabla_{Z} Z, Z\right\rangle=b \lambda^{2}=\frac{1}{2} Z\langle Z, Z\rangle=0 .
$$


It follows that $b=0$, hence $\nabla_{Z} Z=a Z$. But

$$
\left\langle\nabla_{Z} Z, \bar{Z}\right\rangle=Z\langle Z, \bar{Z}\rangle=Z\left(\lambda^{2}\right)=a\langle Z, \bar{Z}\rangle .
$$

It follows that $a=\frac{Z\left(\lambda^{2}\right)}{\lambda^{2}}$, and this proves our claim.

Now, notice that

$$
Z\left(\lambda^{2} H\right)=Z(\langle S \bar{Z}, Z\rangle)=\left\langle\nabla_{Z}(S \bar{Z}), Z\right\rangle+\left\langle S \bar{Z}, \nabla_{Z} Z\right\rangle .
$$

Thus

$$
\begin{aligned}
\left\langle\nabla_{Z}(S \bar{Z}), Z\right\rangle & =Z\left(\lambda^{2}\right) H+\lambda^{2} Z(H)-\left\langle S \bar{Z}, \nabla_{Z} Z\right\rangle \\
& =Z\left(\lambda^{2}\right) H+\lambda^{2} Z(H)-\langle S \bar{Z}, Z\rangle \frac{Z\left(\lambda^{2}\right)}{\lambda^{2}} \\
& =\lambda^{2} Z(H),
\end{aligned}
$$

since $\langle S \bar{Z}, Z\rangle=\lambda^{2} H$.

By using Lemma 5 in Equation (2) we conclude the proof of Proposition 1.

Proof of Theorem 1. By Proposition 1, we have

$$
\begin{aligned}
|\bar{Z} Q(Z, Z)| & =\left|2 \bar{Z}(H) \alpha(Z, Z)+2 \theta \lambda^{2} Z(H)\right| \\
& \leq|d H||\lambda|\left|2 \alpha(Z, Z)+2 \theta \lambda^{2}\right|,
\end{aligned}
$$

where we have used that $|\bar{Z}(H)|=|d H(\bar{Z})| \leq|d H||\bar{Z}|$ and $|Z(H)| \leq$ $|d H||\lambda|$. By hypothesis, $|d H| \leq g\left|Q^{(2,0)}\right|$, for a continuous $g \geq 0$. Thus

$$
\left|\frac{d \psi}{d \bar{z}}\right|=|\bar{Z} Q(Z, Z)| \leq h\left|Q^{(2,0)}\right|=h|\psi(z)|,
$$

where

$$
h=g|\lambda|\left(|\alpha(Z, Z)|+2 \lambda^{2}|H+i \tau|\right),
$$

i.e., $h$ is a continuous nonnegative function on $M$. We now use the Main Lemma of [AdCT] which states that if a function $\psi(z)$ satisfies the inequality (3) in a neighborhood $U$ of a zero $z_{0}$ of $\psi$, then either $\psi \equiv 0$ in a neighborhood $V \subset U$ of $z_{0}$ or, for all $z$ in $V$,

$$
\psi(z)=\left(z-z_{0}\right)^{m} f_{m}(z), \quad m \geq 1, \quad f_{m}\left(z_{0}\right) \neq 0 .
$$


From that we can conclude the proof of Theorem 1 in the same way as in ([AdCT] end of section 2). For completeness, we summarize the argument here.

By the Main Lemma, either $Q^{(2,0)} \equiv 0$, hence by [AR2], $M$ is a surface of revolution, or $Q^{(2,0)}$ has a finite number of zeroes. We show that this case leads to a contradiction. Indeed, the equation $\operatorname{Im}\left[Q(Z, Z) d z^{2}\right]=0$ gives rise to two fields of directions on $M$ whose singularities are the zeroes of $Q(Z, Z)$. The index of any of these fields at the singular point is equal to $-m / 2$, where $m$ is the order of the zero that appears in Equation (4). Since $M$ has genus zero, the sum of the indices of this field of directions, by Poincare Theorem $([\mathrm{H}]$, Chapter III, sect. 2), is two. This is a contradiction, thereby concluding the proof of Theorem 1.

Acknowledgments. This work was done when I. Fernández was visiting IMPA in the second semester of 2005; she wishes to thank people at IMPA for making her stay a pleasurable experience.

\section{References}

[AR1] U. Abresch and H. Rosenberg, A Hopf differential for constant mean curvature surface in $S^{2} \times \mathbb{R}$ and $H^{2} \times \mathbb{R}$. Acta Math. 193 (2004), 141-147.

[AR2] U. Abresch and H. Rosenberg, Generelized Hopf differentials. Matemática Contemporânea, 28 (2005). Sociedade Brasileira de Matemática, 1-28.

[AdCT] H. Alencar, M. do Carmo and R. Tribuzy, A theorem of Hopf and the CauchyRiemann inequality. Preprint, IMPA, 2005. To appear in Comm. in Analysis and Geometry.

[D] B. Daniel, Isometric immersions into 3-dimensional, homogeneous manifolds. Preprint, IMPA, 2005. To appear in Comm. Math. Helv.

[H] H. Hopf, Differential Geometry in the Large. Lectures Notes in Math., SpringerVerlag, volume 1000, 1983. 
Hilário Alencar

Universidade Federal de Alagoas

Instituto de Matemática

57072-900 Maceió, AL

BRASIL

E-mail: hilario@mat.ufal.br

\section{Manfredo do Carmo}

IMPA, Estrada Dona Castorina, 110

22460-320 Rio de Janeiro, RJ

BRASIL

E-mail: manfredo@impa.br

\section{Isabel Fernández}

Depto de Matemática Aplicada I

E.T.S. de Ingenieria Informatica

Universidad de Sevilla

Av. Reina Mercedes s/n

41012 - Sevilla

SPAIN

E-mail: isafer@ugr.es

\section{Renato Tribuzy}

Universidade Federal do Amazonas

Instituto de Ciências Exatas

Departamento de Matemática

Av. Rodrigo Otávio Jordão Ramos, 3000

69077-000 Manaus, AM

BRASIL

E-mail: tribuzy@pesquisador.cnpq.br 